\title{
A quantum mechanical description of the experiment on the observation of gravitationally bound states
}

\author{
A. Westphal \\ Physikalisches Institut der Universität Heidelberg \\ Philosophenweg 12 \\ 69120 Heidelberg, Germany* \\ H. Abele \\ Physikalisches Institut der Universität Heidelberg \\ Philosophenweg 12 \\ 69120 Heidelberg, Germany \\ S. Baeßler \\ Institut für Physik, Universität Mainz \\ Staudinger Weg 7 \\ 55128 Mainz, Germany \\ V.V. Nesvizhevsky and A.K. Petukhov \\ Institut Laue langevin, 6 rue Jules Horowitz, 38042, Grenoble, France \\ K.V. Protasov \\ LPSC, IN2P3-CNRS, UJFG, 53, Avenue des Martyrs, 38026 Grenoble \\ A.Yu. Voronin \\ P.N. Lebedev Physical Institute, \\ 53 Leninsky Prospekt, 119991, Moscow, Russia
}

(Dated: February 10, 2006)

\begin{abstract}
Quantum states in the Earth's gravitational field were observed, when ultra-cold neutrons fall under gravity. The experimental results can be described by the quantum mechanical scattering model as it is presented here. We also discuss other geometries of the experimental setup which correspond to the absence or the reversion of gravity. Since our quantum mechanical model describes, particularly, the experimentally realized situation of reversed gravity quantitatively, we can practically rule out alternative explanations of the quantum states in terms of pure confinement effects.
\end{abstract}

PACS numbers: 03.65.Ge,03.65.Ta,04.62.+v,04.80.-y,61.12.Ex

\section{INTRODUCTION}

A gravitationally bound quantum system has been realized experimentally with ultra-cold neutrons falling under gravity and reflecting off a "neutron mirror" [1, 2]. UCN are neutrons which, in contrast to faster neutrons, are reflected at all angles of incidence. For such UCN, flat surfaces thus act as mirrors. Using an efficient neutron absorber for the removal of higher unwanted states, only neutrons in selected energy states are taken. This idea of observing quantum effects occurring when ultra-cold neutrons are stored on a plane matter surface was first discussed by V.I. Lushikov and I.A. Frank [3] with the first concrete experimental realization in [4]. An experiment in some aspects similar was discussed by $\mathrm{H}$. Wallis et al. [5] in the context of trapping atoms in a gravitational cavity. The toy model of a Schrödinger quantum particle bouncing in a linear gravitational field is known as the quantum bouncer [6, 7]. Retroreflectors for atoms have used the electric dipole force in an evanescent light wave [8, 9] or they are based on the gradient of the magnetic dipole interaction, which has the advantage of not requiring a laser [10].

A unique side-effect of the experiment with neutrons is its sensitivity to gravity-like forces at length scales below $10 \mu \mathrm{m}$ while all electromagnetic effects are extremely suppressed 11, 12. The quantum states probe Newtonian gravity between $10^{-9}$ and $10^{-5} \mathrm{~m}$ and the experiment places limits for gravity-

*Corresponding author. Email address: westphal@sissa.it

Present address: ISAS-SISSA and INFN, Via Beirut 2-4, I-34014 Trieste, Italy 
like forces there. In light of recent theoretical developments in higher-dimensional field theory [13] (see also 14 for explicit realizations in string theory), gauge fields could mediate forces that are $10^{6}$ to $10^{12}$ times stronger than gravity at submillimeter distances, exactly in the interesting range of this experiment and might give a signal in an improved setup.

In this article, we provide the details of a quantum mechanical calculation 15] for our experiment, where gravitational bound quantum states are observed for the first time. The experiment consists of a reflector for neutrons, called neutron mirror, an absorber for unwanted neutrons and a neutron detector. In our previous papers 1, 2, 16] the experiment and a first treatment of the data were presented. Fundamental limits for the spatial resolution and a first ansatz to incorporate the neutron scatterer can be found in [16]. In another work [17] a description of the neutron loss from first principles was developed where the rough edges of the absorber surface are treated as a time-dependent variation of a flat absorber position, modeling the neutron loss mechanism as a process equivalent to the ionization of a particle, initially confined in a well with an oscillating wall. Within the older and more simple model we present in this paper we are able to describe the experimental data with one micro-physical fit parameter which parameterizes the micro-physics of the neutron scatterer/absorber. At the moment our model is the only one yet in which both the data in Fig. 2 and Fig. 4 are described.

The layout of this paper is the following: After describing the experiment and the observations, we recall the quantum mechanics of gravitational bound states on a free mirror, that is without an absorber/scatterer. Then we present an approach to describe the state selection by deriving a neutron loss rate due to non-specular scattering from the rough surface of our absorber. This approach explains the non-classical dependence of the transmission of the mirror-absorber system as a function of the height $l$ of the absorber above the mirror, see Fig. 1 and Fig. 2. We take into account the deformation of the bound state wave functions due to the matter bulk of the state selector, and compare the full prediction with actual data. We also show that the data are only understood when gravity is present.

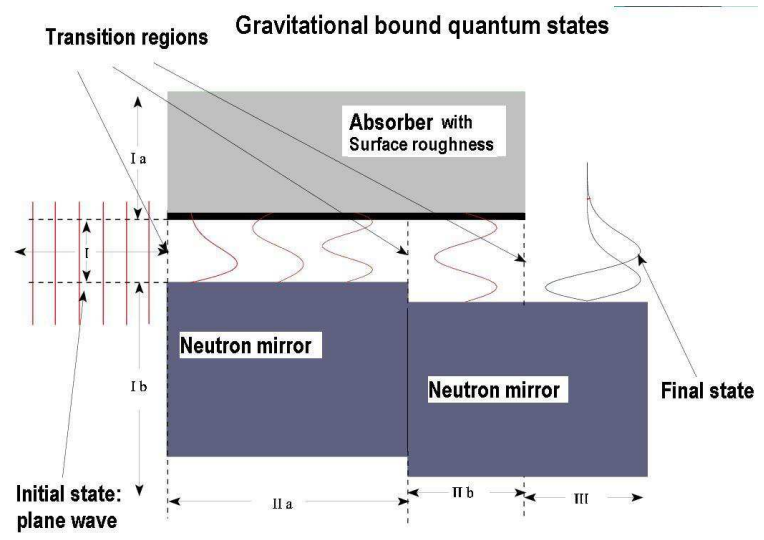

FIG. 1: Schematic view with mirrors, absorber and quantum mechanical boundary conditions. In the experiment, one mirror of length $10 \mathrm{~cm}$ or, as an option as shown here, two bottom mirrors of length $6 \mathrm{~cm}$ were used.

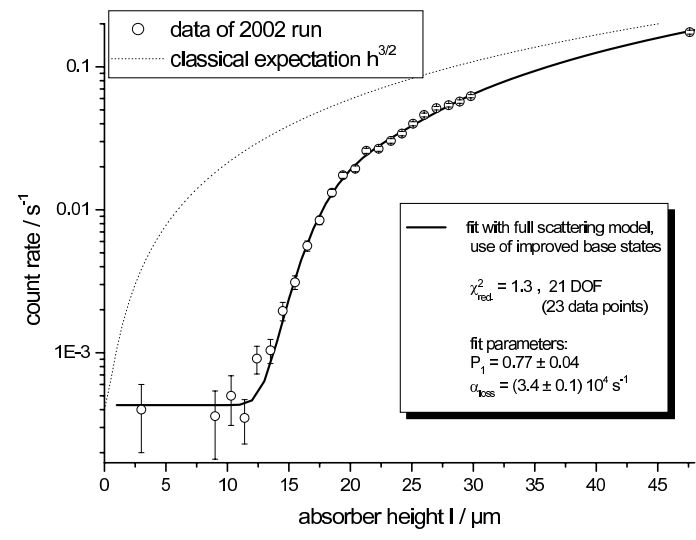

FIG. 2: Circles: Data from the $2^{\text {nd }}$ run 2002 with one bottom mirror [16]. Solid: Transmission coefficient from the phenomenological scattering model. Dash: The classical expectation for the neutron transmission coefficient.

\section{OBSERVATION OF QUANTUM STATES AND SETUP}

A description of the experiment at the Institute Laue-Langevin (ILL) can be found in 2]. It was installed at the UCN facility PF2 of the Institute. Here, neutrons have a velocity of several meters per second. They are then guided to the experiment via a curved neutron guide with a diameter of 80 $\mathrm{mm}$. At the entrance of the experiment, a collimation system cuts down to an adjustable transversal energy $E_{\perp}$ in the pico-eV range. Either one solid block with dimensions $10 \mathrm{~cm} \times 10 \mathrm{~cm} \times 3 \mathrm{~cm}$ or two solid blocks with dimensions $10 \mathrm{~cm} \times 6 \mathrm{~cm} \times 3 \mathrm{~cm}$ composed of optical glass serve as mirrors for UCN neutron reflection. To select different states an absorber/scatterer, a rough mirror coated with an Neutron absorbing alloy, is placed above the first mirror. We can vary the height $l$ above the mirror, that is the size of the slit. The collimation system in front of the state selector is adjusted in such a way that neutrons on classical trajectories entering the experiment have to hit the mirror surface at least two times. After the second mirror we placed a ${ }^{3} \mathrm{He}$ counter for neutron detection. Fig. 1 shows a schematic view of our setup. Signatures of quantum states are observed in the following way: The ${ }^{3} \mathrm{He}$ counter measures the total neutron transmission $F$, when neutrons are traversing the mirror absorber-system 
as described. The transmission is measured as a function of the absorber height $l$ and thus as a function of neutron energy since the height acts as a selector for the energy $E_{\perp}$ of the vertical motion. From the classical point of view, the transmission $F$ of neutrons is proportional to the phase space volume allowed by the absorber. It is governed by a power law $F \sim l^{n}$ and $n=3 / 2$.

The measurements show the following: Above an Absorber/Scatterer height of about $60 \mu \mathrm{m}$, the measured transmission is in agreement with the classical expectation but below $50 \mu \mathrm{m}$, a deviation is clearly visible. Below about $15 \mu \mathrm{m}$, no neutrons can pass the slit. In the next section we will find that the vertical extension of the gravitational bound states increases with the quantum number. Ideally, we expect a stepwise dependence of $F$ as a function of $l$. If $l$ is smaller than the spatial width of the lowest quantum state, then $F$ will be zero. When $l$ is equal to the spatial width of the lowest quantum state $F$ will increase sharply. A further increase in $l$ should not increase $F$ as long as $l$ is smaller than the spatial width of the second quantum state. Then again, $F$ should increase stepwise. At sufficiently high slit width one approaches the classical dependence. Fig. 2 shows details of the quantum regime below an absorber height of $l=50 \mu \mathrm{m}$. The transmission function depends on the horizontal neutron velocity and the absorption efficiency. It was found, that except for the ground state, the stepwise increase is mostly washed out.

\section{QUANTUM MECHANICAL DESCRIPTION OF GRAVITATIONALLY BOUND STATES}

The quantum mechanical treatment of a reflecting neutron mirror, made from glass, is simple. The glass is described by a Fermi pseudo-potential $(V-i W)$. This potential is essentially real $(|W|<<|V|)$ because of the small absorption cross section of glass and $V=100 \mathrm{neV}$ is large compared with the transversal energy $E_{\perp}$ of the neutrons. Therefore, the potential $V$ is set to infinity at height $\mathrm{z}=0$. Neutrons which hit the glass surface undergo specular reflections.

We start with the description of the free states. On a perfect mirror, no mixing of momentum components take place, which leads to a decoupled one-dimensional stationary Schrödinger equation,

$$
\left(-\frac{\hbar^{2}}{2 m} \triangle+V(z)\right) \Psi_{n}=E_{n} \Psi_{n}, V(z)=\left\{\begin{aligned}
m g z & : \quad x \geq 0 \\
\infty & : \quad x<0
\end{aligned}\right.
$$

with wave functions $\Psi_{n}$ for energies $E_{n}$ and the potential $V(z) . m$ is the mass of the neutron and $g$ is the acceleration in the earth's gravitational field. It is convenient to use

$$
\zeta=\frac{z}{R} \text { and, above the mirror, } V=m g R \zeta .
$$

Here, $R$ is a scaling factor, defined as

$$
R=\left(\frac{\hbar^{2}}{2 m^{2} g}\right)^{1 / 3}
$$

Solutions $\Psi_{n, g}(\vec{r}, t)$ of Eq. (1) are obtained with an Airy function

$$
\begin{gathered}
\Psi_{n, g}(\vec{r}, t)=\phi(x, y) \psi_{n, g}(\zeta) e^{-i \frac{E_{n}}{\hbar} t} \\
\psi_{n, g}(\zeta) \quad A i\left(\zeta-\zeta_{n}\right)
\end{gathered}
$$

The displacement $\zeta_{n}$ of the n-th eigenfunction has to coincide with the n-th zero of the Airy function $\left(\operatorname{Ai}\left(-\zeta_{n}\right)=0\right)$ to fulfill the boundary condition $\Psi_{n}(0)=0$ at the mirror. The corresponding energies $E_{n}$ with $z_{n}=R \zeta_{n}$ are

$$
E_{n}=m g z_{n}
$$

In the WKB-approximation we have to leading order

$$
\zeta_{n}=\left(\frac{3 \pi}{2}\left(n-\frac{1}{4}\right)\right)^{2 / 3}
$$

which coincides with the exact eigenvalues to better than $1 \%$ even for the ground state [15]. The $z_{n}$ correspond to the highest point of a classical neutron trajectory with energy $E_{n}$. For example, energies of the lowest levels $(\mathrm{n}=1,2,3,4)$ are $1.44 \mathrm{peV}, 2.53 \mathrm{peV}, 3.42 \mathrm{peV}$ and $4.21 \mathrm{peV}$. The corresponding classical turning points $z_{n}$ are $13.7 \mu \mathrm{m}, 24.1 \mu \mathrm{m}, 32.5 \mu \mathrm{m}$ and $40.1 \mu \mathrm{m}$.

The aim of this experiment was to populate only some of the lowest allowed gravitationally bound quantum states. Higher states were removed with the absorber/scatterer at a certain height $l$. 
It will be most convenient to start with a polychromatic neutron beam of (locally) plane waves entering the system. As is well known, gaussian wave packets being closer to a particle view of a neutron can easily be decomposed into a Fourier integral over plane waves. Using all boundary conditions given by Fig. 1, one arrives at a set of matching conditions [15. The neutron transmission of the system depends both on the eigenvalues and the matching conditions. Furthermore, if two bottom mirrors are used and shifted relative to each other a few $\mu m$ in height (as it was in the 1999 beam time), there is an additional boundary that would change the population of the eigenstates.

The insertion of the states yields a system of linear equations for the matching constants and its solution yields finally the transmission coefficient of the $n^{\text {th }}$ final bound state of region $I I I$. The initial population of the bound states of the wave guide system at coupling-out is uniform, if the vertical velocity distribution of the arriving beam is sufficiently wide and flat - which is of course true in our case, where we have about $20 \mathrm{~cm} / \mathrm{s}$ or $50 \mathrm{peV}$ spread in the vertical components of the arriving beam compared to a few peV for the lowest vertically bound states inside the mirror-absorber system [15] (see also [12]).

In addition, we have to introduce repopulation coefficients $p_{j}$ which allow us to take into account a step between region IIa and IIb into account. If there is no step, all $p_{j}=1$. In the 1999 beam time, the $2^{\text {nd }}$ mirror has been shifted downwards by $5 \mu \mathrm{m}$ relative to the $1^{\text {st }}$ mirror. The matrix of overlap integrals of wave functions at the edge IIa/IIb is sufficiently diagonal that we can neglect the off-diagonal elements. We can set $p_{1}=0.25$ and $p_{j}=1$ for $j>1$. Hence, the relative shift of the bottom mirrors offers a possibility of controlling the relative population of in particular the ground state in the earth's gravitational field. However, even in the setup without step, we find a reduction of the ground state for unknown reasons and keep $p_{1}$ as a free parameter.

In the following, the roughness is described as an additional loss channel in the one-dimensional Schrödinger equation. The neutron loss is then understood in terms of non-specular scattering of neutrons into highly excited states which due to their large vertical energy are rapidly lost inside the glass of the mirror and the absorber/scatterer. Scattering happens at the rough and (due to $V_{\text {absorber }} \simeq 10^{-8} \mathrm{eV} \gg E_{n}$ for states with low $n$ ) hard surface of the absorber, which notion shall enable us to derive the scattering-induced loss rate with just one undetermined micro-physical quantity which would be given in a micro-physical scattering model as a function of the scattering cross section. This parameter will be determined in the end by a fit to the data.

The deformation of the wave functions compared with the purely gravitationally bound states due to the large real part of the absorber potential leads to an approximate vanishing of the bound states at the absorber surface. Therefore, the loss rate is calculated in terms of the deformed states. In a second step, one might model the neutron loss in terms of the physical parameters of the absorber surface but this is outside of the scope of this article.

The neutron removal processes are modeled as a general phenomenological loss rate $\Gamma_{n}(l)$ of the $\mathrm{n}^{\text {th }}$ bound state, which is taken to be proportional to the probability density of the neutrons at the absorber/scatterer. Here $l$ again denotes the position of the absorber/scatterer above the mirror. The modulus of the bound state is then no longer constant in time since it is given as the solution at first order of a differential equation that determines the change of the norm to be proportional to its momentarily value as well as the loss rate

$$
d\left\langle\psi_{n} \mid \psi_{n}\right\rangle=-\left\langle\psi_{n} \mid \psi_{n}\right\rangle \cdot \Gamma_{n}(l) \cdot d t
$$

which yields

$$
\left\langle\psi_{n} \mid \psi_{n}\right\rangle=\left|P_{n}(t)\right|^{2}, \quad P_{n}(t)=e^{-\frac{1}{2} \Gamma_{n}(l) \cdot t} .
$$

The roughness which causes the loss due to scattering can be thought of as being confined to a region of about $2 \cdot \sigma$ width attached to an imagined absorber surface at a height $l$. Here $\sigma$ denotes the rms height roughness of the absorber. Therefore, we give the loss rate of the $\mathrm{n}^{\text {th }}$ bound state in terms of the general description of scattering processes as a function of the probability of neutrons to dwell within the roughness surface region of the absorber/scatterer as

$$
\Gamma_{n}(l)=\alpha_{\mathrm{loss}, \mathrm{n}} \cdot \int_{l-2 \sigma}^{l} d z\left|\psi_{n}(z)\right|^{2} .
$$

Here we used the fact that the geometry of the wave guide system with and without gravity allows for coordinates $(x, y, z)$ with $z$ denoting the transverse coordinate for which the Schrödinger equation 
becomes separable with the product ansatz

$$
\begin{aligned}
\phi_{n}(\vec{r})= & \psi_{n, g}(z) \cdot \phi_{x y}(x, y) \\
& \text { with }: \phi_{x y}(x, y)=\frac{1}{\sqrt{A_{x y}}} \cdot e^{i k_{x} x+i k_{y} y} .
\end{aligned}
$$

Since losses due to non-specular scattering should only depend on local quantities of the surface and the probability of finding neutrons at the surface, we assume that the micro-physics of neutron loss is independent of the 'macro'-physics of the wave function behavior. The above ansatz represents this fact in its product structure which separates the micro-physical quantity $\alpha_{\text {loss,n }}$ completely from the behavior of the wave function described by the probability density $\left|\psi_{n}(z)\right|^{2}$. Therefore, it is the specific loss rate $\alpha_{\text {loss,n }}$ which would depend in a micro-physical model on the roughness properties $\sigma$ (roughness variance) and $\xi$ (roughness correlation length), i.e, $\alpha_{\text {loss,n }}=\alpha_{\text {loss,n }}(\sigma, \xi)$. Furthermore this argument requires that micro-physical quantities should not depend on the neutron state number. Thus, we have $\alpha_{\text {loss,n }}=\alpha_{\text {loss }} \forall n$. Thus, we have

$$
\Gamma_{n}(l)=\alpha_{\mathrm{loss}} \cdot \int_{l-2 \sigma}^{l} d z\left|\psi_{n}(z)\right|^{2} .
$$

We fit this quantity $\alpha_{\text {loss }}$ to the data.

This leaves us with the task to determine the integrals $\int_{l-2 \sigma}^{l} d z\left|\psi_{n}(z)\right|^{2}$ for a state $n$ in a given experimental setup.

\section{A. The wave guide system with gravity}

The bound states $\psi_{n, g}$ in the linear gravitational potential are confined by two very high potential steps above (absorber) and below (mirror). They can be given analytically as:

$$
\psi_{n, g}(z)=A_{n} \cdot A i\left(z / R-\zeta_{n}(l)\right)+B_{n} \cdot B i\left(z / R-\zeta_{n}(l)\right)
$$

and they fulfill the boundary conditions:

$$
\left.\psi_{n, g}\right|_{z=0}=\left.0 \wedge \psi_{n, g}\right|_{z=l}=0
$$

which account for the high potential steps bounding the potential from below and above. Eq.s (12) and (13) together determine the energy eigenvalues $\zeta_{n}(l)$ as functions of the absorber height $l$ which is done numerically.

For a given state $\psi_{n, g}$ the $A i$-part in the wave function is exponentially decaying for $z>z_{n}(l)=$ $\zeta_{n}(l) R$ while the $B i$-part grows exponentially in this region. At $z=l$ both parts add up to zero. As long as $l>z_{n}(l)$ we have $B_{n}<<A_{n}$ because the $B i$-part has to compensate only for the exponentially small value of the $A i$-part at $z=l$. This, however, implies that at $z<l$ we have $\left|B_{n} B i\left(z / R-\zeta_{n}(l)\right)\right|<$ $A_{n} A i\left(z / R-\zeta_{n}(l)\right)$. Therefore, we can ignore the $B i$-part of the wave-function in the calculation of an asymptotic expression for the $\Gamma_{n, g}(l)$ at large $l$. As a result, we have

$$
\psi_{n, g}(z) \approx A_{n} \cdot A i\left(z / R-\zeta_{n}(l)\right)
$$

at $l \gg z_{n}$. The main effect of the $B i$-part is the increase of $\zeta_{n}(l)>z_{n}$ at $l<z_{n}$ where the $z_{n}$ are given by Eq. 6] Here we denote all quantities at $l \rightarrow \infty$ with $\zeta_{n}, A_{n}$ etc. while the corresponding quantities of the realistic states Eq. 12 at finite $l$ are denoted with $\zeta_{n}(l), A_{n}(l)$ etc.

The states $\psi_{n, g}$ are well approximated in the large $l$ regime by the asymptotic WKB states

$$
\begin{array}{r}
\psi_{n, g} \approx \frac{A_{n}}{2 \sqrt{\pi R}} \cdot\left(\zeta-\zeta_{n}\right)^{-1 / 4} \cdot e^{-\frac{2}{3}\left(\zeta-\zeta_{n}\right)^{3 / 2}} \\
\text { if } \zeta>\zeta_{n} .
\end{array}
$$

In the limit $l \rightarrow \infty$ we have the $\zeta_{n}(l) \rightarrow \zeta_{n}$ given by Eq. [6 to $1^{\text {st }}$ order in WKB and $A_{n}=\pi B i\left(-\zeta_{n}\right)$. (For $l \rightarrow \infty$ we have $\psi_{n, g}(z)=A_{n} \cdot A i\left(z / R-\zeta_{n}\right)$. Then the quoted value of $A_{n}$ which is exact ensures that $\left\langle\psi_{n, g} \mid \psi_{n, g}\right\rangle=1$.) In the case $l=R \zeta_{l} \lesssim z_{n}$ consider the WKB-expression for the energy eigenvalues

$$
\int_{0}^{\zeta_{l}<\zeta_{n}(l)} d \zeta \sqrt{\zeta_{n}(l)-\zeta}=\pi(n-1 / 4)
$$




$$
\int_{0}^{\zeta_{l}<\zeta_{n}(l)} d \zeta \sqrt{\zeta_{n}(l)-\zeta}=\zeta_{l} \sqrt{\zeta_{n}(l)}+\mathcal{O}\left(\frac{\zeta_{l}^{2}}{\zeta_{n}^{2}(l)}\right)
$$

we arrive at

$$
\zeta_{n}(l)=\frac{\pi^{2}(n-1 / 4)^{2}}{\zeta_{l}^{2}}
$$

which coincides with the known box state expression for large $n$.

Now plugging the asymptotics of the gravitationally bound states Eq. 15 into Eq. 11] one arrives at a prediction for the loss rate that reads

$$
\begin{aligned}
\Gamma_{n, g}(l) & =\alpha_{\text {loss }} \cdot \int_{l-2 \sigma}^{l} d z \cdot\left|\psi_{n, g}(z)\right|^{2} \\
& \simeq \alpha_{\text {loss }} \cdot \frac{\sigma}{2 \pi R} \cdot\left|A_{n}\right|^{2} \cdot \frac{e^{-\frac{4}{3}\left(\frac{l-z_{n}}{R}\right)^{3 / 2}}}{\sqrt{\left(l-z_{n}\right) / R}} \quad \text { for } l \gg z_{n} .
\end{aligned}
$$

Now look at the behavior of the total neutron flux $F$ through the wave guide if a large number of states contribute to it (semi-classical limit). We have $F=\int_{\mathcal{A}} j_{x} \sim \sum_{n}\left\langle\psi_{n} \mid \psi_{n}\right\rangle$ where we get $\left\langle\psi_{n} \mid \psi_{n}\right\rangle$ from Eq. 8 and $\mathcal{A}$ denotes the wave guide cross section. If gravity is present the behavior $\Gamma_{\text {loss }} \sim$ $\exp \left(-4 / 3 \cdot\left(\zeta-\zeta_{n}(l)\right)^{3 / 2}\right)$ leads to the fact that each time when $l \simeq z_{n}=R \zeta_{n}$ a new state rapidly starts to contribute to the transmission. Therefore at a given large height $l$ the number of states contributing to $\phi(l)$ reads from Eq. 6

$$
l=z_{n} \sim N^{2 / 3} \Rightarrow N(l) \sim l^{3 / 2}
$$

which yields asymptotically (if $N(l)$ large and thus $\Delta N_{N-(N-1)} / N=1 / N \rightarrow 0$ ) the classical behavior in a gravitational field. A simple phase space argument [2] shows that a perfect absorber at the top in presence of a gravitational field yields a classical transmission

$$
F(l) \sim l^{3 / 2} .
$$

\section{B. A wave-guide system without gravity}

Now we can use the model to derive the loss rate for bound states in the absence of gravity. These bound states are well approximated by those which describe the quantum dynamics of a particle in a one-dimensional box with infinitely high walls, i.e. the so-called box states. They are given by

$$
\psi_{n}=\sqrt{\frac{2}{l}} \cdot \sin \left(\frac{n \pi}{l} \cdot z\right)
$$

which yields a loss rate given by

$$
\begin{aligned}
\Gamma_{n}(l) & =\alpha_{\mathrm{loss}} \cdot \int_{l-2 \sigma}^{l} d z \cdot\left|\psi_{n}(z)\right|^{2} \\
& =\alpha_{\mathrm{loss}} \cdot\left[\frac{2 \sigma}{l}-\frac{1}{2 n \pi} \sin \left(\frac{4 n \pi}{l} \cdot \sigma\right)\right] .
\end{aligned}
$$

A comparison of the full box state expression (line 2 in the above equation) with the numerical result for the loss rate with gravity using the full states Eq. 12 is given graphically in Fig. [3]

The exact expression in the second line of Eq. 21 approaches a constant for $n \rightarrow \infty$ rendering the sum in Eq. 23] divergent. Therefore, in a realistic fit we have to include the fact that in any real experiment the number of box states $N$ in the wave guide is finite. Firstly, the collimator system in front of the wave guide yields an input vertical velocity distribution of finite width. Secondly, for wider vertical velocity spectra all neutrons with $v_{z}>v_{z}^{\text {crit }}=4.3 \cdot \mathrm{m} \cdot \mathrm{s}^{-1}$, the critical velocity of glass, will enter the mirror or the absorber directly without forming bound states in the wave guide. In both cases the number of box states which is populated by the entering flux of neutrons behaves like $N \sim l$. If the collimator is tuned to yield an input vertical velocity distribution of small width (i.e. about $10 \mathrm{~cm} / \mathrm{s}$ ) we 
have $N / l \sim 2 \cdot \mu m^{-1}$ corresponding to about $N \approx 200$ box states in a wave guide of $l \sim 100 \mu m$ width. Thus, we have evaluated a finite sum with $N / l=2 \cdot \mu m^{-1}$ when comparing the gravity-free prediction of Eq. 23 with the experimental data sets (for $l<100 \mu m$ we have $N<200$ box states populated with $N$ approaching $\approx 200$ for $l \rightarrow 100 \mu \mathrm{m})$. The dependence of our result on the choice of the cutoff $N$ is very weak. If the critical velocity of glass defines the cutoff this results in $N / l \sim 20 \cdot \mu m^{-1}$ (corresponding to about $N \approx 2 \cdot 10^{3}$ box states in a wave guide of $l \sim 100 \mu \mathrm{m}$ width). We find that the predictions agree for both cutoff choices with each other to far better than the experimental accuracy within the $l$-range of the measurement.

Now in the absence of gravity the asymptotic behavior of the transmitted flux $F(l)$ carried by the box states at large $l$ can be given directly from the Eq.s 8 and 21 For $n \sigma / l \ll 1$ we can approximate Eq. 21] with

$$
\Gamma_{n}(l)=\alpha_{\text {loss }} \cdot 16 \pi^{2} / 3 \cdot \sigma^{3} \cdot \frac{n^{2}}{l^{3}} \quad \text { if : } \quad \frac{n \sigma}{l} \ll 1 .
$$

If the approximation in Eq. 22 were valid for all $n$ we would have

$$
\begin{aligned}
F(l) & \sim \sum_{n \geq 1} e^{-\Gamma_{n}(l) \cdot t_{\text {flight }}} \\
& =\sum_{n \geq 1} e^{-\gamma \cdot n^{2}}, \text { with }: \gamma=16 \pi^{2} / 3 \cdot \sigma^{3} \cdot l^{-3} \cdot t_{\text {flight }} \\
& =\frac{1}{2} \cdot\left(\vartheta_{3}\left(0, e^{-\gamma}\right)-1\right) \\
& \sim l^{3 / 2} \quad \text { for large } l .
\end{aligned}
$$

Here $\vartheta_{n}(q, u)$ denotes the elliptic theta function where we used Mathematica [18] to evaluate the sum. This result differs from the naive classical behavior of a gravity-free wave guide with a perfect absorber: In the case of the linear trajectories describing classical particles in absence of the gravitational field, we find 15

$$
F(l) \sim l^{2}
$$

which is easy to imagine since one factor of $l$ obviously has its origin in the relation $F(l) \sim \mathcal{A} \sim l$ while the $2^{\text {nd }}$ factor encodes that the range of vertical velocities $\Delta v_{z}$ of particles which pass the wave guide without ever touching the absorber also behaves like $\Delta v_{z} \sim l$.

Finally, from this situation we expect in general an interpolating behavior of the gravity-free transmission rate with respect to its power-law dependence on the absorber height. In fact, if the gravityfree prediction of Eq. 23 is carried out using the exact expression for the loss rates $\Gamma_{n}$ in Eq. 21 for $N / l>200 \cdot \mu m^{-1}$, i.e. $N>2 \cdot 10^{4}$ box states at $l \approx 100 \mu m$, we find that the transmission begins to deviate from an $l^{3 / 2}$-power law towards an $l^{n}$-dependence with $n \rightarrow 2$ which is the general dependence to be expected both classically and quantum mechanically. Thus, the behavior of the gravity-free prediction as $F(l) \sim l^{3 / 2}$ in our given experimental situation is an artifact caused by the relatively small number of box states $\left(N \lesssim 2 \cdot 10^{3}\right.$ for $\left.l \leq 100 \cdot \mu m\right)$ in the wave guide.

\section{Reversed geometry}

We now turn to the third case of $g \rightarrow-g$ instead of $g \rightarrow 0$. This inversion of gravity is equivalent to a setup geometry where the absorber/scatterer is placed at the bottom at $z=0$ and a movable mirror at $z=l$ above the absorber. For this situation we can follow the derivation of Subsection IVA Since the absorber is now at $z=0$ we have to evaluate the probability integral at this position which implies for large $l$ the use of the asymptotic WKB expression

$$
\begin{array}{r}
\psi_{n, g} \approx \frac{A_{n}}{\sqrt{\pi R}} \cdot\left(\zeta_{n}-\zeta\right)^{-1 / 4} \cdot \sin \left[\frac{2}{3}\left(\zeta_{n}-\zeta\right)^{3 / 2}+\frac{\pi}{4}\right] \\
\text { if } \zeta<\zeta_{n} .
\end{array}
$$

This results in

$$
\Gamma_{\text {loss }}^{(n, g, \text { rev. })}(l)=\alpha_{\text {loss }} \cdot \frac{\sigma}{2 \pi R} \cdot\left|A_{n}\right|^{2} \cdot \frac{16}{3} \sqrt{\frac{z_{n}}{R}} \cdot \frac{\sigma^{2}}{R^{2}}
$$


Note, that this loss rate of the reversed geometry is practically independent on the state number $n$ since $\lim _{n \rightarrow \infty}\left|A_{n}\right|^{2} \sqrt{\zeta_{n}}=\pi$ and even at $n=1$ it is $\left|A_{1}\right|^{2} \sqrt{\zeta_{1}} / \pi-1 \lesssim 0.5 \%$. Comparing this result with the corresponding expression Eq. 19] for the normal geometry we find that the ratio of the fluxes $F_{n}^{(g) \text {,rev. }}(l) / F_{n}^{(g)}(l)$ of the $n^{\text {th }}$ state for $l>z_{n}$ between the reversed and the normal geometry is given by

$$
\frac{F_{n}^{(g), \text { rev }}(l)}{F_{n}^{(g)}(l)}=e^{-\alpha_{\text {loss }} \cdot \frac{\sigma}{2 \pi R} \cdot\left|A_{n}\right|^{2} \cdot \frac{16}{3} \sqrt{\frac{z_{n}}{R}} \cdot \frac{\sigma^{2}}{R^{2}} \cdot \frac{L}{v_{h o r .}}} .
$$

Since $\frac{\sigma}{2 \pi R} \cdot\left|A_{1}\right|^{2} \cdot \frac{16}{3} \sqrt{\frac{z_{1}}{R}} \cdot \frac{\sigma^{2}}{R^{2}} \cdot \frac{L}{v_{\text {hor. }}} \approx 7.2 \cdot 10^{-5} \mathrm{~s}$ for experimental values of $L=0.13 \cdot \mathrm{m}, v_{\text {hor. }} \approx 10 \cdot \mathrm{m} / \mathrm{s}$ and $\sigma=0.75 \mu \mathrm{m}$ a value of $\alpha_{\text {loss }} \gtrsim \mathcal{O}\left(10^{4} s^{-1}\right)$ fitted from a measurement of the normal geometry would result in a huge asymmetry under a $\pi$-rotation around the optical axis of the wave guide when comparing the normal and the reversed geometry.

\section{A FIT TO THE DATA}

For a comparison with the measurements we plug the resulting loss rates into the general prediction for the transmitted neutron flux Eq. 28 Together with the repopulation coefficient $p_{1}$ accounting for eventual shifts between split bottom mirrors one predicts

$$
F(l)=F_{0}+\sum_{n} F_{n}(l)=F_{0}+C \cdot\left\{p_{1} \cdot e^{-\Gamma_{\text {loss }}^{(1)}(l) \cdot \frac{L}{v_{h o r .}}}+\sum_{n>1} \cdot e^{-\Gamma_{\text {loss }}^{(n)}(l) \cdot \frac{L}{v_{h o r .}}}\right\}
$$

$F_{0}$ is the detector background and $C$ the total flux normalization. $\alpha_{\text {loss }}$ is the universal parameter introduced above which parameterizes the scatterer strength. This quantity is in general expected to be a rather weak function of the roughness parameters $\sigma$ and $\xi$ which would be determined in principle by a microphysical calculation of the loss due to non-specular scattering at the rough absorber surface. Thus it does not depend on the absence or presence of gravity nor on the state number $n$.

In the case of $l \lesssim z_{n}$, the absorber/scatterer will begin to squeeze the bound states once they start to 'feel' it sufficiently strongly. This implies further that due to $E_{n, g}(l) \geq E_{n, g}(\infty)=E_{n, g}$ pure gravity for the true bound states a sufficiently small $l$ leads to $E_{n, g}(l) \gg m g \cdot l$. Therefore, the calculation of the $\Gamma_{\text {loss }}^{(n)}$ is done using the full realistic bound states Eq. 12 and deriving the relations corresponding to Eq.s 19] 21] and 26] numerically which incorporates the mentioned behavior.

\section{A. Normal geometry}

The detector background $\phi_{0}$ has been measured independently to yield $F_{0}=(0.0043 \pm 0.0004) s^{-1}$ for the 1999 measurement [1] and $F_{0}=(0.0004 \pm 0.0001) s^{-1}$ for the new 2002 measurement using an improved setup 16]. Thus, one remains with having to determine the two universal quantities $\alpha_{\text {loss }}$ and $C$ from the data. $C$ turns out to be completely fixed by the data points at $l>70 \mu m$, and thus also has been measured. The fit therefore will be a 1-parameter one determining $\alpha_{\text {loss }}$ as long as all the populations $p_{n}$ stay to be equal.

We fit now Eq. 28 to the newer data from the run of the experiment in 2002 which has a different absorber and better statistics, and systematic effects are smaller than in 1999. The results are shown in Fig. 2. The value of $\alpha_{\text {loss }}$ is found in a fit to the data. The result of the fit yields $(L=13 \mathrm{~cm}$, $\left.v_{\text {hor. }} \approx 5 \cdot m \cdot s^{-1}\right)$

$$
\alpha_{\text {loss }}=(3.4 \pm 0.1) \cdot 10^{4} s^{-1}
$$

The fit was done using neutrons with only one value of the horizontal velocity which was chosen to be the average velocity $v_{h o r} \approx 5 \cdot m \cdot s^{-1}$. This approximation produces essentially the same results as if one uses the full actual spectrum of horizontal velocities produced by the collimating system to calculate the transmission.

The 2002 run was performed with only one bottom mirror so that no significant repopulation effects of the ground state are expected. However, it was necessary to allow the population of the ground state 
to shift towards $75 \%$ compared to the excited states in order to describe the data: $p_{1}=0.77 \pm 0.04$. This suppression is significant, but it is not large leaving all the states still to be approximately equally populated.

In the measurement of the 1999 run, two bottom mirrors were used. It was tried to shift these mirrors relative to each other vertically and in alignment by a few micrometers. Small shifts of a few microns between two bottom mirrors change the population of the ground state and the next state quite drastically: A shifted geometry with a relative mirror shift of about $5 \mu \mathrm{m}$ and no relative tilt of the two bottom mirrors results in $p_{1} \approx 0.25$ compared to $p_{n}=1, n \geq 2$. The fit to the data (see Fig. 4) results in $\alpha_{\text {loss }}=(5.3 \pm 0.5) \cdot 10^{4} s^{-1}$ and $p_{1}=0.24 \pm 0.1$ which would be consistent with the possible relative mirror shift discussed above.

\section{B. Reversed geometry}

In a second setup described by the term 'reversed geometry', the absorber was placed at the bottom and the mirror above. Here, the position where the scattering-inducing roughness is found at $z=0$. This case can also be derived from the states Eq. 12 by just placing the roughness appropriately on bottom and then calculating the corresponding loss rates. The bound states are now again given by the Airy functions. However, at $z=0$ where the absorber is now placed, they do not decay exponentially fast and the gravitationally bound states will be strongly absorbed at arbitrary heights $l$ of the mirror at top - quite contrary to the normal setup. If gravity were absent, such a $\pi$-turn of the wave guide around its optical axis would not have such an effect. Thus, there would be no preferred direction in space forcing the transmission factor to be invariant under rotations around the optical axis of the system since the box states describing the simple confinement situation without gravity are symmetric with respect to the optical axis. This check was done with the 1999 data. We took the prediction for gravitationally bound states of Eq. 12 in the normal setup and fitted $\alpha_{\text {loss }}$ and $p_{1}$ to the data. The model yields $p_{1}=0.24 \pm 0.1$ and $\alpha_{\text {loss }}=(5.3 \pm 0.5) \cdot 10^{4} s^{-1}$. Taking now these values for $\alpha_{\text {loss }}$ and $p_{1}$ from the fit, we can calculate a prediction for the setup with reversed geometry using the results of Subsection IVC - which is now entirely fixed and not fitted any more. Using the experimental setup parameters used there, $L=13 \mathrm{~cm}$ and $v_{\text {hor. }} \approx 10 \cdot \mathrm{m} / \mathrm{s}$ we can estimate the suppression factor using Eq. 27. on the ground state which yields

$$
\frac{F_{1}^{(g), \text { rev. }}(l)}{F_{1}^{(g)}(l)} \approx 0.03 .
$$

This fits well with the two data points obtained experimentally in this reversed setup. Using the full prediction calculated again numerically from Eq. 12] in the reversed setup, the comparison with the 1999 data is shown in Fig. 4 In treating the 1999 data other models [2, 11] showed more pronounced steps. These steps are not present in the (more advanced) model presented in this paper.

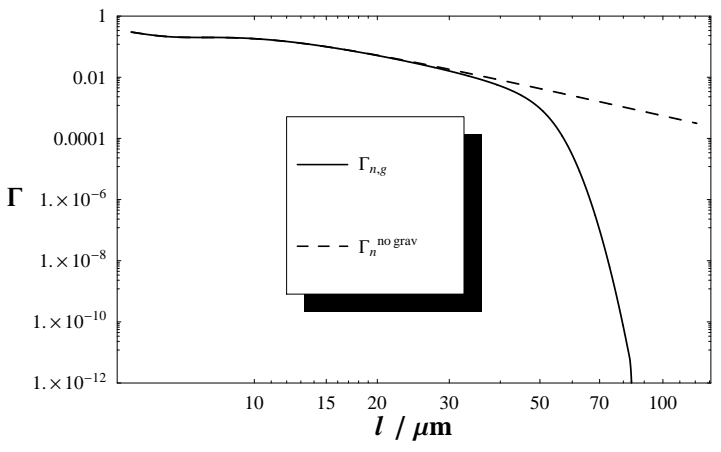

FIG. 3: Behavior of the loss rate $\Gamma$ (plotted in arbitrary units) for the pure box state $n=5$ (dash), an approximate power law, and for the gravitationally bound state $n=5$ found numerically from using the full states Eq. 12 (solid), showing exponentially fast decay above some $l=\mathcal{O}(R)$.

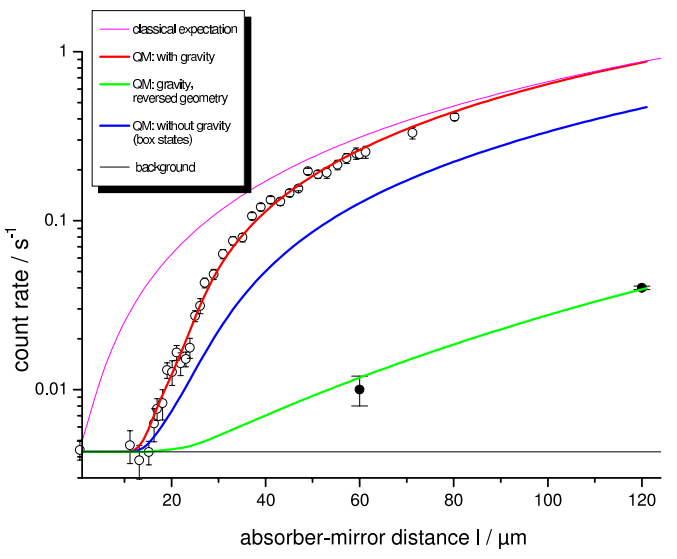

FIG. 4: Open Circles: Transmission as a function of the absorber-mirror distance. Filled Circles: Transmission measured with inverse geometry - the absorber on the bottom and a $10 \mathrm{~cm}$ mirror on top (both are 1999 measurement data [2]).

The above described asymmetry of the neutron transmission of a wave guide with one absorbing 
and one reflecting wall under rotations around its optical axis in the presence of gravity has indeed been measured and can be described quantitatively. This rules out the possibility to explain the data just by confinement effects in terms of box states inside a rectangular box-shaped potential [19] and thus establishes the gravitational nature of force which binds the neutron bound states to the bottom mirror. For further discussion, see also [20]. The measurement of the reverse geometry is a very good test of our (or probably any) model, since the result depends strongly on the absorber properties. The fact that the absorber model which describes the data in the normal geometry can also be used for the reversed geometry, without any new fit parameters, gives us confidence that our model is a useful tool to describe the data.

\section{SUMMARY}

For the first time the existence of quantized bound states of neutrons in the gravitational field of the earth above a horizontal glass mirror was experimentally demonstrated. Here we present a quantum mechanical model providing an accurate fit to the data. The difficult part is the incorporation of the neutron absorber into this model. We show that we can describe its action with only one phenomenological fit parameter in several configurations. The most important configuration has bound quantum states in the linear gravitational potential. We consider repopulation effects of these states when a step from a second mirror is present. The emerging reduction of the first state has been calculated. The standard setup uses only one mirror. Also in this case, the data show an unexpected reduction of the first quantum state as well.

Further, it is shown that the experiment would generate different results, if gravity were hypothetically to be turned off. This configuration is a wave-guide system with box states which describe the quantized motion of a particle a one-dimensional box with infinitely high walls.

A striking difference is found, if the gravitational field changes sign. This last case has been observed by our measurement [1] and can be described quantitatively within the same modeling of the absorber mechanism presented here. Thus, the dominating effect of gravity on the formation of the bound states has been demonstrated since the measurement did not show just a simple confinement effect, as proposed in [19]. On the contrary, it could not be described theoretically, if the earth's gravitational field was not present.

\section{REFERENCES}

[1] V. V. Nesvizhevsky et al., Nature 415, 297 (2002).

[2] V. V. Nesvizhevsky et al., Phys. Rev. D 67, 102002 (2003) arXiv:hep-ph/0306198.

[3] V. I. Luschikov and A. I. Frank, JETP Lett. 28559 (1978).

[4] V. V. Nesvizhevsky et al., Nucl. Instrum. Meth. A 440, 754 (2000).

[5] H. Wallis et. al., Appl. Phys. B 54407 (1992).

[6] R. L. Gibbs, Am. J. Phys. 4325 (1975).

[7] H. C. Rosu, arXiv:quant-ph/0104003.

[8] C. G. Aminoff et al.,, Phys. Rev. Lett. 713083 (1993).

[9] M. A. Kasevich, D. S. Weiss, and S. Chu, Opt. Commun. 15607 (1990).

[10] T. M. Roach et al., Phys. Rev. Lett. 75629 (1995).

[11] H. Abele, S. Baeßler and A. Westphal, Lect. Notes Phys. 631, 355 (2003) arXiv:hep-ph/0301145.

[12] V. V. Nesvizhevsky and K. V. Protasov, Class. Quant. Grav. 21, 4557 (2004) arXiv:hep-ph/0401179.

[13] N. Arkani-Hamed, S. Dimopoulos and G. R. Dvali, Phys. Lett. B 429, 263 (1998) arXiv:hep-ph/9803315; I. Antoniadis, N. Arkani-Hamed, S. Dimopoulos and G. R. Dvali, Phys. Lett. B 436, 257 (1998) arXiv:hep-ph/9804398;

N. Arkani-Hamed, S. Dimopoulos and G. R. Dvali, Phys. Rev. D 59, 086004 (1999) arXiv:hep-ph/9807344; I. Antoniadis, Lect. Notes Phys. 631, 337 (2003).

[14] D. Cremades, L. E. Ibanez and F. Marchesano, Nucl. Phys. B 643, 93 (2002) arXiv:hep-th/0205074; C. Kokorelis, Nucl. Phys. B 677, 115 (2004) arXiv:hep-th/0207234.

[15] A. Westphal, Diploma thesis, Institute of Physics, University of Heidelberg (2001) arXiv:gr-qc/0208062.

[16] V. V. Nesvizhevsky et al., Eur. Phys. J. C 40, 479 (2005) arXiv:hep-ph/0502081.

[17] A. Yu. Voronin et al., arXiv:quant-ph/0512129.

[18] S. Wolfram, The Mathematica Book, 4th Edition, Cambridge Univ. Press, 1999.

[19] J. Hansson, D. Olevik, C. Turk and H. Wiklund, Phys. Rev. D 68, 108701 (2003) arXiv:quant-ph/0308108. 
[20] V. V. Nesvizhevsky et al., Phys. Rev. D 68, 108702 (2003). 